\title{
On one Approach for Stable Estimate of Technical System Efficiency
}

\author{
Sharif E. Guseynov 1, 2, a, Alexander I. Urbah ${ }^{2, \text { b }}$, Sergey A. Andreyev ${ }^{2, ~ c ~}$ \\ ${ }^{1}$ Institute of Mathematical Sciences and Information Technologies, Liepaja University, \\ 14 Liela Street, Liepaja LV-3401, Latvia; \\ ${ }^{2}$ Institute of Aeronautics, Faculty of Transport and Mechanical Engineering, \\ Riga Technical University, \\ 1 A Lomonosov Street, Building-1, Riga LV-1019, Latvia \\ Sh.E.Guseinov@inbox.lv, ${ }^{b}$ Aleksandrs.Urbahs@rtu.lv, ${ }^{c}$ S.A.Andreyev@gmail.com
}

\begin{abstract}
In present paper the problem of efficiency evaluation of technical system by measurable structural design parameters is investigated. To accomplish the purpose of considered problem the mathematical model is constructed in the form of a finite-dimensional operator equation, where desired elements are both influence weights of the calculated structural design parameters and technical effectiveness indicator of the system. First, the constructed model is reduced to the normal system, and then the apparatus of the ill-posed inverse problem theory is used for the reduced problem: a regularizing operator is constructed and an algorithm for finding the regularization parameter is developed.
\end{abstract}

Keywords: Efficiency evaluation of technical systems, ill-posed and inverse problems, mathematical model, regularizing algorithm.

\section{INTRODUCTION}

The technical system according to one of numerous definitions (see, for instance, [1], [2]) is a set of elements of the system (mechanisms, assemblies, components and similar components of the system) that interact with each other during the process of performing the specified functions. In the named definition it is worth to emphasize that the concepts "the technical system" and "the system components" can be mutually expressed one by the other, and depending on the purpose of the research, the required accuracy, the information level about the reliability and the similar factors and characteristics, the considered / introduced concrete concept "the technical system" for the certain problem-target can be converted into "the system element" for another problem-target. However, in contrast to the above given definition of the technical system, in this paper we distinct the "system" and "elements" concepts and we try to identify, first, the level of influence (in other words: the importance / significance / weight / weight of influence) of possible defects in each element of the technical system on the operability of all other elements (with or without possible defects in these elements) of the same technical system; second, the resulting / cumulative impact of possible defects in all elements of the given technical system on the operation of the whole investigated technical system.
Here, anticipating things, it is necessary to note the following:

- if such mutual influence of possible defects in the elements of the technical system are missing or negligible then the investigation results of the constructed mathematical model, which describes the considering problem of the defects mutual influence, should reflect this fact;

- the technical system possible defects in all elements resulting / cumulative influence on the operating of the whole studied technical system is not a simple sum of the effects of these defects, and, moreover, may not be a linear combination (with constant coefficients) of the effects of these defects.

The aforementioned considerations lead us to the following two natural questions:

1. What is the measure of influence of each element possible defects of the given technical system on each of all the other technical system elements performance? Is it possible to define these individual measures of defects mutual influence in cases when there is only given a set of calculated / controllable parameters measurement values of the system?

2. If the answer to the first question, which has been formulated above, is positive, then is it 
possible to stably (in the sense of Tikhonov: see, for instance, [3]) find out the effectiveness of the technical system under investigation without any additional assumptions and information?

Before attempting to answer these two questions, let us briefly discuss the general scheme of the technical systems effectiveness quantitative evaluation. It should be emphasized that speaking about the technical system effectiveness usually is meant the effectiveness of its use as an active mean for typical operations, for which purposes the system is intended. If the considered technical system is a multi-purpose, and if such a multipurpose technical system for each objective has a high efficiency, it means that such a system has wide functionality, i.e. it has qualities that reflect the ability of the system to meet the challenges associated with achieving each goal. In applied researches, the effectiveness of technical systems usually has to solve the following two problems: (1) the problem of choice, which essence is to evaluate operations effectiveness with the use of technical system; (2) the problem of choice, which essence lies in the choice of a rational strategy for the use of technology in the operation. The problem of effectiveness is closely related to the reliability problem in technical systems see, for instance, [4], [5]). The complexity growth of technical systems reduces their reliability and, therefore, reduces their effectiveness. Unreliable technical system cannot be an effective way of achieving the goal. The operation effectiveness analysis provides an approach to determine the required parameters of the technical system reliability that are used as active means in the operation. This is one of the important aspects of the reliability and effectiveness problems interconnection. Another aspect of the relationship of these problems is to determine the rational ways of use for the available resources to improve the reliability of the developing technical systems.

The essence of this work - to give an unequivocal answer for the formulated above two important issues without requiring additional information about the technical state of system elements. As it will be shown the obtaining of such a clear answer is done by transformation of the technical system efficiency indicator finding task to the inverse problem, which has the form of the first kind finite-dimensional operator equation, and a subsequent development of a regularized method for its sustainable solutions.

\section{GENERAL SCHEME OF EFFICIENCY EVALUATION OF TECHNICAL SYSTEMS AND METHODS OF WEIGHTING COEFFICIENTS}

There are many methods for the quantitative evaluation of the effectiveness of technical systems (see, for instance, [1], [4], [5]), the majority of which are operation research methods: the criterion of efficiency and constraints (significant and sign constraints) are made; the output effect and costs (or the result of the operation of the technical system) are determined, wherein, the costs can be determined at the production or design stages of the considered technical system, as well as during operation of the system. However, all these methods have a common scheme, which can be represented as the functional

$$
E[y ; t] \stackrel{\text { def }}{\equiv} F\left(y_{1}, \ldots, y_{m} ; C\right)(t),
$$

where $m \in \mathbb{N}$ is a number of elements in the technical system; $y_{i}=f_{i}\left(x_{1}, \ldots, x_{n} ; t\right)$ is a technical indicator of the system $i$-th $(i=\overline{1, m})$ element, which is also called the $i$-th partial quality indicator; $n \in \mathbb{N}$ is a number of controllable / calculated (mainly structuralconstructive) parameters of the technical system; $\left\{x_{j}\right\}_{j=\overline{1, n}}$ are controllable / calculated parameters of the system; $t$ is time; $C$ is total expenses (in other words, the total cost) for the development, design and operation of the system.

As is clear from (1), the variation in methods for finding the efficiency $E[y ; t]$ is caused by specific types of functions $f_{i}\left(x_{1}, \ldots, x_{n} ; t\right)(i=\overline{1, m})$ and $F$. Namely, constructing the function $f_{i}\left(x_{1}, \ldots, x_{n} ; t\right)(i=\overline{1, m})$ and $F$ in different ways, we obtain different formulas for calculating $E$, at the same time, if designed function $f_{i}\left(x_{1}, \ldots, x_{n} ; t\right)(i=\overline{1, m})$ and $F$ will have an analytical form, then the calculation of $E[y ; t]$ is not difficult, since the controllable / calculated parameters of the investigated technical system are known for each case / state of the system; if the explicit form of the functions is not known, then in order to calculate $E[y ; t]$ are mainly used statistical methods or sometimes only evaluation of $E[y ; t]$ according to one of the most important quality partial indicators $y_{i m p} .(\operatorname{imp} \in\{1 ; \ldots ; m\})$, and over the other partial quality indicators are placed constraints so that they do not exceed certain limits:

$$
\left\{\begin{array}{l}
E[y ; t]=y_{\text {imp }}, \\
\underline{y_{i}} \leq y_{i} \leq \overline{y_{i}}(i=\overline{1, m} /\{i m p\}),
\end{array}\right.
$$

where $\underline{y_{i}}$ and $\overline{y_{i}}$ are respectively the lower and upper limits of $i$-th $(i=\overline{1, m})$ partial quality indicator.

The effectiveness evaluation of the technical systems according to the rule (2) has the disadvantage - the solution of the corresponding optimization problem (i.e., the same problem (2) with the criterion $E[y ; t]=y_{i m p}$. $\rightarrow$ sup or the problem of implementation choice for practical optimization technical system version will be ambiguous, since the 
criterion choice is ambiguous: you can get a lot of options for systems with the same or almost the same main partial quality indicator $y_{\text {imp }}$. with significantly different other partial indicators that satisfy the constraints; therefore, it cannot be guaranteed that the determined version of the system will be closer to the optimum.

One of the widely used and well-studied methods for the effectiveness estimation of technical systems is the method of weighting coefficients, in which the function $F$ is represented as a linear combination of functions $y_{i}(i=\overline{1, m})$ :

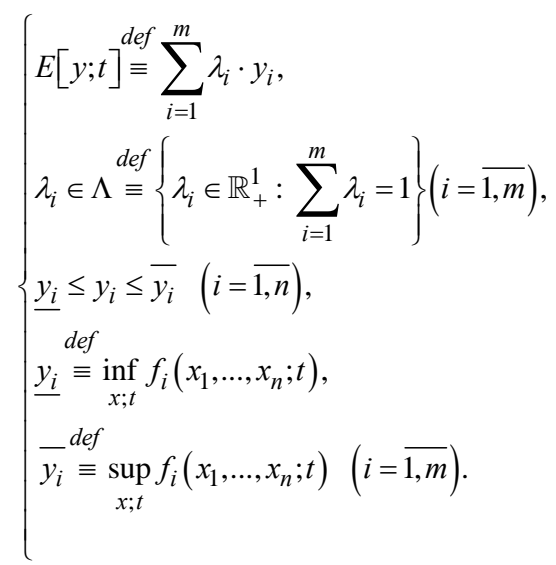

In (3) weighting coefficient $\lambda_{i} \in \Lambda$ is called the coefficient importance / significance of the $i$-th $(i=\overline{1, m})$ partial quality indicator and generally speaking, is the desired number; set $\Lambda$ is called the set of importance or preference. Without going into details, we list the well-known, the main advantages and disadvantages of the method of weighting coefficients (3), the final destination of which is the choice of the best option among the alternatives in the development, design or operation of complex technical systems. As the merits may be listed the following: ease of formalization; the possibility of taking into account all the main partial quality indicators; opportunity to take into account the preferences of the decision maker about the problem during the weighting coefficients setup; clear physical / technical / economic sense. The main drawbacks the following may be indicated: subjectivity in weighting coefficients; the accountability lack of dependence on the weighting coefficients values of partial quality indicators.

In conclusion of this section we note that the independent objectives are both determination of the weighting coefficients of partial quality indicators of the technical system and the problem of finding / evaluation of these partial system indicators. Currently, there are a lot of analytical, analytical and numerical, logical, statistical, sets and graphs based, expert, etc. methods, which are successfully used, for solving these independent problems. Exhaustive information about the basic and the most common these methods, as well as a fairly complete overview of them with reasonable instructions of strengths, weaknesses and areas of application can be found in [5], [6]. Exhaustive information on the design of experiments in technics / technology and processing of the obtaining experimental data can be found in [7][9]. Therefore, in this paper we will not deal with any problem of finding controllable / calculated structural design parameters $\left\{x_{j}\right\}_{j=\overline{1, n}}$ of technical system, or partial quality indicators estimation problem $\left\{y_{i}\right\}_{i=1, m}$ of technical elements of the system, and in the following sections of this paper, we assume that all of them are known to us aposteriori.

\section{MATHEMATICAL FORMULATION OF THE PROBLEM}

We consider the functional

$$
E[y ; t] \equiv \sum_{i=1}^{\text {def }} \lambda_{i} \cdot y_{i},
$$

appearing in the method of weighting coefficients (3). Obviously, the formula (4) to calculate the efficiency of technical systems from the known information about the partial quality indicators $y_{i}(i=\overline{1, n})$ of system elements is unable to determine which of the controllable/calculated structural design parameters of technical system in a given period of time has a significant impact on partial indicators of the elements of the technical system and, consequently, the common efficiency indicator of the system. Furthermore, according to this formula it is impossible to determine how different is the influence of the same structural design parameters to different parts of the system, which partial quality indicators (consequently, their reliability) significantly differ from each other or, conversely, very similar. In other words, the formula (4) does not distinguish the influence measure of each controlled parameter of the system on common efficiency indicator of the system, both in time and in the given set of controlled technical parameters for each element of the system. Therefore, the partition of the elements of technical system under consideration by their influence both on the partial quality indicators of elements, and the common efficiency of the whole system is impossible. Obviously, the disadvantage of the formula (4) is removed if for each element of the system we will take into account each controllable technical parameter with its "individual influence" the weight, which will depend on both the time $t$ and on each controlled/calculated indicator (index $j$ ) of each element (index $i$ ) of considered technical system. In other words, instead of the method (3) we propose to consider the method (let us call it the method of individual weighting coefficients), in which 
the functional of efficiency indicator of technical system has the form

$$
E[i ; t] \stackrel{d e f}{\equiv} C_{1} \cdot \sum_{j=1}^{n} w_{i j}(t) \cdot \hat{x}_{i j}(t)+C_{2},
$$

where $n \in \mathbb{N}$ is a number of controllable/calculated structural design parameters of technical system; $m \in \mathbb{N}$ is the elements number of the technical system; for each fixed $i(i=1, \bar{m})$ and $t \in\left[T_{\text {start }}, T_{\text {end }}\right]$ the parameters $\quad\left\{\hat{x}_{i j}(t), j=\overline{1, n}\right\} \quad$ is a result of standardization by means of any method applied to initial controllable structurally design parameters of technical system, for example, by the formula (for instance, see the fundamental books [10], [11]) $\hat{x}_{i j}(t)=\left(x_{i j}(t)-m_{j}\right) / \sigma_{j}$, through $m_{j}$ is denoted the average deviation of the $j$-th indicator and by $\sigma_{j}$ is denoted the standard deviation of the $j$-th indicator; $\left[T_{\text {start }}, T_{\text {end }}\right]$ is time interval during which the technical system effectiveness is studied and the structural design parameters values of technical system are fixed (locked); $T_{\text {start }}$ and $T_{\text {end }}$ are respectively the initial and final moments of the time interval; constants $C_{i}(i=1 ; 2)$ are chosen in such a way that they are, respectively, the centers of two clusters of technical system elements with the worst and the best values of structural and design parameters, wherein, if the clusters overlap, it is possible to apply FCM (the fuzzy c-means clustering algorithm) or PCM (the possibilistic c-means algorithm) algorithm (see, for instance, [12]); for each $i$-th $(i=1, \bar{m})$ element of technical system the desired weight of the $j$-th $(j=1, \bar{n})$ controllable / calculated structural design parameter into the common effectiveness indicator of the entire technical system at every fixed time $t \in\left[T_{\text {start }}, T_{\text {end }}\right]$ denoted as $\left\{w_{i j}\right\}_{i=\overline{1, m}}^{j=\overline{1, n}}$. Coefficients $\left\{w_{i j}\right\}_{i=1, m}^{j=1, n}$ we call the weights of individual influence.

Thus, in the proposed formula (5) the numbers $n \in \mathbb{N}, \quad m \in \mathbb{N}, \quad T_{\text {start }} \in \mathbb{R}_{+}^{1}, \quad T_{\text {end }} \in \mathbb{R}_{++}^{1}$, $\left\{\hat{x}_{i j}(t) \in \mathbb{R}_{+}^{1}\right\}_{i=1, m}^{j=1, n} \quad$ and $\quad C_{i} \in \mathbb{R}^{1}(i=1 ; 2)$ are known data; the weight of individual influence $\left\{w_{i j}\right\}_{i=1, m}^{j=\overline{1, n}}$ and the common effectiveness indicator $E[i ; t]$ of technical system are sought. It is obvious that in formula (5) there are exactly $m \cdot(n+1) \cdot T_{s \rightarrow e}$ (here $T_{s \rightarrow e}$ means the length of the time interval $\left[T_{\text {start }}, T_{\text {end }}\right]$ measured in an integer number of conventional units) unknown $w_{i j}(t)$ and $E(i, t)$, which should be uniquely defined via $\left(m \cdot n \cdot T_{s \rightarrow e}+2\right)$ initial data $x_{i j}(t), A, B$, connected by exactly $m \cdot T_{s \rightarrow e}$ linear algebraic equations.

Indeed, the individual influence coefficient $\left\{w_{i j}\right\}_{i=\overline{1, m}}^{j=\overline{1, n}}$ are time-dependent, i.e. $w_{i j}=w_{i j}(t)$ for $\forall i \in\{1,2, \ldots, m\}$ and $\forall j \in\{1,2, \ldots, n\}$, since the influence measure of the same structural design parameter of the considered technical system on the partial component of the same element of the technical system at different time may vary. In other words, generally speaking, $w_{i_{f i x} j_{f i x}}\left(t_{1}\right) \neq w_{i_{f i x} j_{f i x}}\left(t_{2}\right)$ for $t_{1} \neq t_{2}$. However, taking in formula (5) the stationary weights in the individual influence, we are thus considering the case when the weights are different only relatively $n$ controlled / calculated structural design parameters of technical system, and are constants in time $t \in\left[T_{\text {start }}, T_{\text {end }}\right]$ and relatively elements $i=\overline{1, m}$. Therefore, the introduced functional (5) under these assumptions is equivalent to functional $E[i ; t]=C_{1} \cdot \sum_{j=1}^{n} w_{j} \cdot \hat{x}_{i j}(t)+C_{2}$. Further, for the time interval $\left[T_{\text {start }}, T_{\text {end }}\right]$ constructing a discrete mesh in the form of $t_{k} \in\left[T_{\text {start }}, T_{\text {end }}\right]: \quad t_{k}=T_{\text {start }}+(k-1) \cdot \tau$, $k=\overline{1, K} ; \quad \tau=\left(T_{\text {end }}-T_{\text {start }}\right) /(K-1) ; \quad K \in \mathbb{N} /\{1\}$, and then introducing the notation $\hat{x}_{i k j} \equiv \hat{x}_{i j}\left(t_{k}\right)$ and $E_{i k} \equiv E\left[i ; t_{k}\right]$ for $i=\overline{1, m} ; j=\overline{1, n} ; k=\overline{1, K}$; we obtain the following final analytical formula for the functional in the proposed method of the individual weighting coefficients:

$$
E_{i k}=C_{1} \cdot \sum_{j=1}^{n} w_{j} \cdot \hat{x}_{i k j}+C_{2} .
$$

So, summarizing the abovementioned and abstracting from the subject area of the considered problem, we can formulate the following mathematical problem: it is required to find a stable solution of a linear algebraic equations system (6) relatively $(m \cdot K+n)$ unknown $\left\{E_{i k}\right\}_{i=\overline{1, m}}^{k=\overline{1, K}}$ and $\left\{w_{j}\right\}_{j=1, n}$ for $(n \cdot m \cdot K+5)$ known initial data $\left\{\hat{x}_{i k j} \in \mathbb{R}_{+}^{1}\right\}(i=\overline{1, m} ; k=\overline{1, K} ; j=\overline{1, n}) ; \quad n \in \mathbb{N} ; \quad m \in \mathbb{N} ;$ $K \in \mathbb{N} /\{1\} ; \quad C_{i} \in \mathbb{R}^{1}(i=1 ; 2)$. Obviously, the system (6) is not a normal system, and therefore, the concept of its "solution" should be clarified. 
IV

\section{REDUCTION OF THE PROBLEM TO THE INVERSE PROBLEM}

Let us introduce an augmented matrix $\tilde{X}$ with dimensions $(m \cdot K) \times(m \cdot K+n)$, which of elements $\left\{\tilde{x}_{i k j}\right\}$ are calculated by the following rule:

$$
\tilde{x}_{i k j} \stackrel{\text { def }}{\equiv}\left\{\begin{array}{l}
-1 \text { if }(j>n) \wedge(K \cdot i+k=j+(K-n)) ; \\
0 \text { if }(j>n) \wedge(K \cdot i+k \neq j+(K-n)) ; \\
C_{1} \cdot \hat{x}_{i k j} \text { if } j \leq n .
\end{array}\right.
$$

Then the system (6) has the form:

$$
\sum_{j=1}^{m \cdot K+n} \tilde{x}_{i k j} \cdot \tilde{w}_{j}=-C_{2} \forall(i=\overline{1, m} ; k=\overline{1, K}),
$$

where

$$
\tilde{w}_{j} \stackrel{\text { def }}{\equiv}\left\{\begin{array}{l}
w_{j} \text { if } j \leq n ; \\
E_{i k} \text { if } j=n+(i-1) \cdot K+k, i=\overline{1, m}, k=\overline{1, K} .
\end{array}\right.
$$

Introducing the notation $\quad \tilde{W} \equiv\left\{\tilde{w}_{j}\right\}_{j=1,(m \cdot K+n)}$; $\tilde{C} \equiv(\underbrace{-C_{2}, \ldots,-C_{2}}_{m \cdot K})^{T}$, the system (8) becomes

$$
\tilde{X} \tilde{W}=\tilde{C} .
$$

Thus, the original problem (6) is reduced to the problem (9), where we want to define the $(m \cdot K+n) \times 1$ size vector $\tilde{W}$ under the known $(m \cdot K) \times(m \cdot K+n)$ size matrix $\tilde{X}$ and the $(m \cdot K) \times 1$ size vector $\tilde{C}$. The system (9) consists of $m \cdot K$ equations with $(m \cdot K+n)$ unknown $\tilde{w}_{j}$, i.e. number of unknown is larger exactly by $n$ values than the amount of equations. In other words, the system (9) is an underdetermined system of linear algebraic equations. Hence, the question arises: what should we mean by a solution of the system (9)? We introduce the following concept.

Definition 1. Let us call the element $\tilde{W}$ as a generalized solution of underdetermined system (9) if

$$
\tilde{W}_{g . s}=\arg \min _{\tilde{W} \in \mathbb{R}^{m \cdot K+n}}\|\tilde{X} \tilde{W}-\tilde{C}\|_{\mathbb{R}^{m \cdot K}}^{2},
$$

where the norm in a Euclidean space $R^{m \cdot K}$ is understood as a Schur norm: $\|y\|_{\mathbb{R}^{m \cdot K}}=\sqrt{\sum_{j=1}^{m \cdot K} y_{i j}^{2}}$.

It is obvious that in order to find the generalized solutions of the system (9), according to the definition 1 , it is required to solve the unconditional optimization problem $\|\tilde{X} \tilde{W}-\tilde{C}\|_{\mathbb{R}^{m \cdot K}}^{2} \rightarrow \min _{\tilde{W} \in \mathbb{R}^{m \cdot K+n}}$. For this purpose, we construct the Euler equation (for instance, see [3]) for the functional $V(\tilde{W}) \stackrel{d e f}{\equiv}\|\tilde{X} \tilde{W}-\tilde{C}\|_{\mathbb{R}^{m \cdot K}}^{2}$ and then we equate it to zero: $\delta V \stackrel{\text { def }}{=} 2 \cdot\left\langle\Delta W, \tilde{X}^{T} \tilde{X} \tilde{W}+\frac{1}{2} \cdot \tilde{X}^{T} \tilde{X} \Delta W-\tilde{X}^{T} \tilde{C}\right\rangle=0$. Hence, due to the arbitrariness of the increment $\Delta W$, we will have

$$
\tilde{X}^{T} \tilde{X} \tilde{W}=\tilde{X}^{T} \tilde{C},
$$

The finite-dimensional operator equation (11) is the Euler equation for the unconditional extremum problem $\|\tilde{X} \tilde{W}-\tilde{C}\|_{\mathbb{R}^{m \cdot K}}^{2} \rightarrow \min _{\tilde{W} \in \mathbb{R}^{m \cdot K+n}}$. The operator equation (11), in contrast to the system (9), is a normal system: there are exactly $(m \cdot K+n)$ linear algebraic equations and the same number of unknowns $\left\{\tilde{w}_{j}\right\}_{j=1,(m \cdot K+n)}$. So, we see that the generalized solution $\tilde{W}_{g . s}$ of the system (9) is a classical solution of the normal system of linear algebraic equations (11), and vice versa. Since the matrix $\tilde{X}$, elements of which are calculated by the formula (7), is strongly sparse matrix, the determinant of the principal matrix $\tilde{X}^{T} \tilde{X}$ can be arbitrarily small, i.e. $\operatorname{det}\left(\tilde{X}^{T} \tilde{X}\right) \ll 1$ or even $\operatorname{det}\left(\tilde{X}^{T} \tilde{X}\right)=0$. Consequently, it is wrong to solve the system (11) by any direct methods, i.e. the desired solution $\tilde{W}$ of the system (11) cannot be expressed by the formula $\tilde{W}=\left(\tilde{X}^{T} \tilde{X}\right)^{-1} \tilde{X}^{T} \tilde{C}$, from which according to the well-known Cauchy inequality $\|\tilde{W}\| \leq\left\|\left(\tilde{X}^{T} \tilde{X}\right)^{-1} \tilde{X}^{T}\right\| \cdot\|\tilde{C}\| \quad$ implies stability of the problem (9) solution by $\tilde{C}$ and $\tilde{X}$ in the sense of a generalized solution. In addition to the abovementioned problem of ill-conditioned system (11) (this causes the instability of its solution), violation of the condition $\operatorname{det}\left(\tilde{X}^{T} \tilde{X}\right) \neq 0$ also violates the Hadamard correctness (see, for instance, [3], [13], [14]) of the system (9) in the sense of a generalized solution, namely, the generalized solution, as the solution of the normal system (11), may be nonunique, and then is determined up to elements of the kernel $\operatorname{ker} \tilde{X}$ of main matrix $\tilde{X}$. Therefore, in this case if $\tilde{W}^{(1)}$ is a generalized solution of the system (9), then each column-vector $\tilde{W}=\tilde{W}^{(1)}+\tilde{W}^{(2)}$, where $\tilde{W}^{(2)}$ is an element of the kernel $\operatorname{ker} \tilde{X}$, i.e. $\tilde{W}^{(2)} \in \operatorname{ker} \tilde{X}$, will also be a generalized solution of the system (9), moreover, all these solutions can arbitrarily differ from each other. Therefore, there should be formulated solution selection criterion. The following concept gives the criterion:

Definition 2. The generalized solution with minimal Schur norm we call the normal generalized solution. In addition, the pseudosolution of the system $A z=u\left(z \in \mathbb{R}^{n} ; u \in \mathbb{R}^{m} ; A=\left\{a_{i j}\right\}_{i=1, \overline{1, n}}^{\overline{\overline{1}} \overline{\mathrm{n}}}\right) \quad$ is called (the 
pseudosolution concept first introduced in the works [15], [16]; a vector $z^{\text {(pseudo) }} \in \mathbb{R}^{n}$, which minimizes the residual $\|A z-u\|$ on the entire space $\mathbb{R}^{n}$. Since the system $A z=u$ may have more than one pseudosolution, then we can talk about the set $F_{A}$ of pseudosolutions $A z=u$. The normal solution of the system $A z=u$ respecting to the vector $z^{(1)} \in \mathbb{R}^{n}$ is called (for instance, see [3]) a pseudosolution $z^{(0)} \in \mathbb{R}^{n}$ with minimal norm $\left\|z-z^{(1)}\right\|$, i.e. such that $\left\|z^{(0)}-z^{(1)}\right\|=\inf _{z \in F_{A}}\left\|z-z^{(1)}\right\|$. For simplicity we can always assume that $z^{(1)} \equiv 0$, and then the normal solution with respect to vector $z^{(1)} \in \mathbb{R}^{n}$ can be simply called the normal solution. It is important to emphasize that for any system $A z=u$ the normal solution always exists and is unique. It is appropriate to note that the normal solution of the system $A z=u$ can also be defined as a pseudo-solution that minimizes a given positive definite quadratic form in the coordinates of the vector $z-z^{(1)}$. Since the set of generalized solutions $\left\{\tilde{W}_{g . s} \in \mathbb{R}^{m \cdot K+n}:\left\|\tilde{X} \tilde{W}_{g . s}-\tilde{C}\right\|_{\mathbb{R}^{m \cdot K}}^{2}=\min _{W \in \mathbb{R}^{m \cdot K}}\|\tilde{X} \tilde{W}-\tilde{C}\|_{\mathbb{R}^{m \cdot K}}^{2}\right\}$ is a compact set (i.e. convex and closed set), then by the Weierstrass theorem, there exists a unique element $\tilde{W}_{g . s}$ of this compact set, which has the least distance from zero, i.e. there exists a unique element $\tilde{W}_{g . s}$ with the smallest norm. In other words, the normal generalized solution is unique, and it is uniquely determined. It is known, such a solution of the system (9) can be found using the pseudoinverse MoorePenrose matrix: $\tilde{W}_{g . s}=\tilde{X}^{+} \tilde{C}$, where through $\tilde{X}^{+}$is denoted the pseudoinverse Moore-Penrose matrix with the dimensions $(m \cdot K+n) \times(m \cdot K)$, and is given by

$$
\tilde{X} \tilde{X}^{+} \tilde{X}=\tilde{X}
$$

or by theoretical asymptotic formula

$$
\tilde{X}^{+}=\lim _{\varepsilon \rightarrow 0}\left(\tilde{X}^{T} \tilde{X}+\varepsilon \cdot I\right)^{-1} \tilde{X}^{T},
$$

where through $I$ is denoted an identity matrix.

It should be emphasized that both the relation (12) and the asymptotic formula (13) are inconvenient for practical finding of $\tilde{X}^{+}$. Relatively easy formula for finding practical pseudoinverse matrix $\tilde{X}^{+}$is the following formula:

$$
\tilde{X}^{+}=\tilde{X}_{1}^{+} \tilde{X}_{2}^{+}=\tilde{X}_{1}^{T}\left(\tilde{X}_{1} \tilde{X}_{1}^{T}\right)^{-1}\left(\tilde{X}_{2} \tilde{X}_{2}^{T}\right)^{-1} \tilde{X}_{2}^{T},
$$

where $\tilde{X}=\tilde{X}_{1} \tilde{X}_{2}$ is an ambiguous skeleton decomposition of the matrix $\tilde{X}$, at that $\tilde{X}_{2}$ has the dimension $(m \cdot K) \times r$, but $\tilde{X}_{1}$ has the dimension $r \times(m \cdot K+n)$, where $r=\operatorname{rang} \tilde{X}=\operatorname{rang} \tilde{X}_{1}=\operatorname{rang} \tilde{X}_{2}$. At different skeletal decompositions we always obtain the same normal generalized solution for pseudoinverse matrix $\tilde{X}^{+}$.

Now let us ask whether the normal generalized solution is stable? Since the normal generalized solutions $\tilde{W}_{g . s}$ satisfies the inequality $\left\|\tilde{W}_{g . s}\right\| \leq\left\|\tilde{X}^{+}\right\| \cdot\|\tilde{C}\|$, then it is obvious, the normal generalized solution is stable for variations of right side of the system (9). However, the construction of pseudoinverse matrix $\tilde{X}^{+}$by formulas (12)-(14) implies that the solution (9) in the sense of the normal generalized solution does not become stable for arbitrary variations of the main matrix $\tilde{X}$ of system (9). Therefore, if the elements of matrix $\tilde{X}$ initially and/or during the computer calculations may start perturbing (for studied in this paper problem such disturbances are quite typical, because the original data $\quad \tilde{x}_{i k j}(i=\overline{1, m} ; j=\overline{1, n} ; k=\overline{1, K}), \quad$ calculated according to the formula (7), are the results of experimental measurements of controlled/calculated structural design parameters of considered technical system, and the measurement results always have errors), then the method of Moore-Penrose does not give a true solution of the system (9). Consequently, the problem of finding a stable solution of a finite dimensional operator equation (11), and thus, a stable solution of the system (9), is still open, and it is required to construct the regularized algorithms for the normal generalized solution finding. Note that if the equation (11) is solvable in a classical way, then its normal generalized solution is called a normal solution.

Thus, the obtained problem (11) refers to the theory of inverse problems. As already mentioned above, we cannot take every found element $\tilde{W}$ from (11) as an exact or approximate solution. It is intuitively clear that there is needed the possible solutions selection principle. It usually requires the use any available additional information about the solution. Such information may have qualitative or quantitative nature, and the desire to use quantitative information allows select a compact set $M \subset F$, where initially Hadamard ill-posed problem becomes well-posed problem (according to Tikhonov). In other words, the use of additional quantitative information eliminates the instability of the inverse problem (11) solution.

\section{THE CONSTRUCTION OF THE REGULARIZED SOLUTION ALGORITHM}

Thus we consider the problem

$$
\tilde{X}^{T} \tilde{X} \tilde{W}=\tilde{X}^{T} \tilde{C}, \quad \tilde{W} \in F ; \tilde{X}^{T} \tilde{C} \in U,
$$

where the inverse operator $\left(\tilde{X}^{T} \tilde{X}\right)^{-1}$ does not exist, or exists, but, generally speaking, is not a continuous 
operator on the image of the set $F$ when mapping it using the operator $\tilde{X}^{T} \tilde{X}$, i.e. on the set of $\tilde{X}^{T} \tilde{X} F$. Furthermore, the problem (15) is essentially incorrect, i.e. in the equation (15) small change of the right-hand side $\tilde{X}^{T} \tilde{C} \in U$, which is related to its approximate nature, may lead outside the set $\tilde{X}^{T} \tilde{X} F$.

Thus, in (15) the set $F$ of possible solutions of the equation (15) is not compact. Suppose that it is a priori known that the function $\tilde{W}_{e x}$ is a normal generalized solution of equation (15) at the precisely given right-hand side part $\tilde{X}^{T} \tilde{C}=\left(\tilde{X}^{T} \tilde{C}\right)_{e x}$, i.e. $\tilde{X}^{T} \tilde{X} \tilde{W}_{e x}=\left(\tilde{X}^{T} \tilde{C}\right)_{e x}$. If in (15) instead of the precisely given right-hand side part $\left(\tilde{X}^{T} \tilde{C}\right)_{e x}$ we know its approximation $\left(\tilde{X}^{T} \tilde{C}\right)_{\delta}$, as well as the value $\delta$, that characterizes the closeness degree of $\left(\tilde{X}^{T} \tilde{C}\right)_{\delta}$ to $\left(\tilde{X}^{T} \tilde{C}\right)_{e x}$, the problem is to find an approximate normal generalized solution $\tilde{W}_{\delta}$ to the exact solution $\tilde{W}_{e x}$, having the stability towards small changes $\left(\tilde{X}^{T} \tilde{C}\right)_{\delta}$ property by the known initial data $\left\{\left(\tilde{X}^{T} \tilde{C}\right)_{\delta} ; \delta\right\}$. As it has been already mentioned in the previous section of this work, for an approximation of the normal generalized solution $\tilde{W}_{\delta}$ of equation (15) we cannot use the exact solution of the approximate equation $\tilde{X}^{T} \tilde{X} \tilde{W}_{\delta}=\left(\tilde{X}^{T} \tilde{C}\right)_{\delta}$. In other words, the vector $\tilde{W}_{\delta}=\left(\tilde{X}^{T} \tilde{X}\right)^{-1}\left(\tilde{X}^{T} \tilde{C}\right)_{\delta}$ is not an approximate normal generalized solution of equation (15) with the approximate right-hand side $\quad \tilde{X}^{T} \tilde{C}=\left(\tilde{X}^{T} \tilde{C}\right)_{\delta}$ : $\rho_{U}\left(\left(\tilde{X}^{T} \tilde{C}\right)_{e x},\left(\tilde{X}^{T} \tilde{C}\right)_{\delta}\right) \leq \delta$, since the vector $\tilde{W}_{\delta}$ exists for not any arbitrary element $\tilde{X}^{T} \tilde{C} \in U$. Furthermore, as it was shown in the previous section of this work, the normal generalized solution lacks stability property to small changes in the right hand side $\tilde{X}^{T} \tilde{C}$ of equation (15). The value $\delta \geq \rho_{U}\left(\left(\tilde{X}^{T} \tilde{C}\right)_{e x},\left(\tilde{X}^{T} \tilde{C}\right)_{\delta}\right)$ characterizes the error of the equation right hand side part of (15). Therefore, obviously, it is necessary to determine an approximate normal generalized solution $\tilde{W}_{\delta}$ of equation (15) using an operator (action/technique), which depends on the numerical parameter $\delta \geq \rho_{U}\left(\left(\tilde{X}^{T} \tilde{C}\right)_{e x},\left(\tilde{X}^{T} \tilde{C}\right)_{\delta}\right)$. And the values of this operator must be taken according to the error $\delta$, namely, the consistency must be such that the approaching in the metric space $U$ of the right hand side part $\left(\tilde{X}^{T} \tilde{C}\right)_{\delta}$ of equation (15) to the exact value $\left(\tilde{X}^{T} \tilde{C}\right)_{e x}$, the found (by means of an operator, which depends on a parameter $\delta$ ) approximated normal generalized solution $\tilde{W}_{\delta}$ approaches to the desired exact normal generalized solution $\tilde{W}_{e x}$ of the equation $\tilde{X}^{T} \tilde{X} \tilde{W}_{e x}=\left(\tilde{X}^{T} \tilde{C}\right)_{e x}$ in the Schur metric $F$. Thus, the main task - the problem of the equation approximate normal generalized solution finding (15), resistant to minor changes in the right-hand side part, - reduces to the solution of the following two "side" problems: the first "side" problem is to find a regularizing operator for the equation (15) relatively to the element $\left(\tilde{X}^{T} \tilde{C}\right)_{e x}=\tilde{X}^{T} \tilde{X} \tilde{W}_{e x}$; the second "side" problem is to determine the regularization parameter $\alpha$ by additional information about the problem, for example, the error value, which is given for the righthand side $\left(\tilde{X}^{T} \tilde{C}\right)_{\delta}$ (in this case $\alpha=\alpha(\delta)$ ).

Let us discuss now these "sides" problem. First of all we assume that a regularizing operator exists: we denote it by $R=\left\{r_{k m}\right\}$. Then, as a solution to the original problem (15) the element $\tilde{W}=R \tilde{X}^{T} \tilde{C}$ may be chosen. Let us construct a regularizing operator $R$. For this purpose the algorithm, enables finding the inverse $\left(\tilde{X}^{T} \tilde{X}\right)^{-1}$ matrix, is based on the regularization method idea by academician A.Tikhonov ([3]). Another regularization algorithm, which has proved itself to be suitable enough while solving various both linear finite-dimensional operator equations of a first kind, and linear infinite-dimensional operator equations of a first kind is presented below. The algorithm consists of the following steps:

Step 0 . The initial data are given $n \in \mathbb{N} ; \quad m \in \mathbb{N}$; $\left\{\hat{x}_{i k j} \in \mathbb{R}_{+}^{1}\right\}(i=\overline{1, m} ; k=\overline{1, K} ; j=\overline{1, n}) ; \quad C_{i} \in \mathbb{R}^{1}(i=1 ; 2)$ in equation system (6). (15).

Step 1 . The origin system (6) is reduced to a system

Step 2. The sequence of parameters, for example, $\alpha_{l}=2^{-l} \quad(l=1,2,3, \ldots)$, is taken and for the two (eg., the first two) adjacent parameters $\alpha=\alpha_{l_{0}}$ and $\alpha=\alpha_{l_{0}+1}$ of this sequence is solved the following system equations relatively to $(m \cdot K+n)^{2}$ unknowns $r_{l i}^{\alpha}$ :

$$
\alpha \cdot r_{l i}^{\alpha}+\sum_{j=1}^{m \cdot K+n} r_{l k}^{\alpha} \cdot \beta_{j i}=\left(\tilde{X}^{T} \tilde{X}\right)_{i l} \quad \forall(i, l=\overline{1,(m \cdot K+n)}),
$$


where $\beta_{j i} \equiv \sum_{p=1}^{\operatorname{def}}\left(\tilde{X}^{T} \tilde{X}\right)_{j p} \cdot\left(\tilde{X}^{T} \tilde{X}\right)_{i p}$.

Step 3. For each of the parameters $\alpha=\alpha_{l_{0}}$ and $\alpha=\alpha_{l_{0}+1}$ the coordinates $\left\{\tilde{w}_{l}^{\alpha}\right\}_{l=1, \overline{1(m \cdot K+n)}}$ of the column-vector $\tilde{W}^{\alpha}$ are found by formula

$$
\tilde{w}_{l}^{\alpha}=\sum_{i=1}^{m \cdot K+n} r_{l i}^{\alpha} \cdot\left(\tilde{X}^{T} \tilde{B}\right)_{i} \quad(l=\overline{1,(m \cdot K+n)}) .
$$

Step 4. The condition $\left\|\tilde{W}^{\alpha_{l_{0}}}-\tilde{W}^{\alpha_{l_{0}+1}}\right\|_{\mathbb{R}^{m \cdot K+n}} \leq \varepsilon_{0}$ is verified, where $\varepsilon_{0}$ is the desired accuracy (for example, $\varepsilon_{0}=10^{-3}$ ) of the approximate normal generalized solution regarding the exact solution (which is unknown). If the answer is positive, then we finish the algorithm: the approximate normal generalized solution $\tilde{W}^{\alpha_{1}} \approx \tilde{W}^{\alpha_{2}}$ is found and, therefore, the desired $w_{j}(j=\overline{1, n})$ and $\left\{E_{i k}\right\}_{i=1, m}^{k=\overline{1, K}}$ in the initial equation (6) are found, wherein we have $w_{j}=\tilde{w}_{j}$ for $j \leq n$, and for $\forall(i=\overline{1, m} ; k=\overline{1, K})$ such that $j=n+(i-1) \cdot K+k$, we have $E_{i k}=\tilde{w}_{j}$ for $j=\overline{(n+1),(m \cdot K+n)}$. If $\left\|\tilde{W}^{\alpha_{l_{0}}}-\tilde{W}^{\alpha_{l_{0}+1}}\right\|_{\mathbb{R}^{m \cdot K+n}}>\varepsilon_{0}$, then we move to the step 2, where we assume $\alpha_{l_{0}}=\alpha_{l_{0}+1}$ and $\alpha_{l_{0}+1}=\alpha_{l_{0}+2}$. The given theoretical reasoning of the papers [17]-[19] guarantees the algorithm is finite, and the result of the algorithm will be a normal generalized solution of the system (6).

Remark. The desired optimal regularization parameter $\alpha=\alpha_{\text {opt }}$ can be found by means of qualitatively different method - generalized residual method (see, for instance, see [20]), which is more versatile and accurate method, but also requires more complex calculations. A qualitatively new approach to find quasi-optimal regularization parameter, which combines simplicity and accuracy, is in detail described (with many appendices) in the monographs [13], [14]).

\section{CONCLUSIONS}

In this paper we investigate the problem of the effectiveness estimation of the technical systems from the measurable structural design parameters. A fundamentally new approach, where, in contrast to the traditional approaches, is no need to determine in advance the kind of partial quality indicators of the technical system and to find the weights of these partial indicators (the weights determination of particular indicators is subjective and ambiguous: hence, it cannot be guaranteed to identify which option of the destination choice weights provides optimal effectiveness of the studied technical system). The proposed in this paper approach is based on the apparatus of the inverse and ill-posed problem theory, namely, first, a mathematical model is built, which is the underdetermined finite-dimensional operator equation with respect to unknown influence weights of the calculated structural design parameters, as well as the desired effectiveness indicator of the technical system; the built model is reduced in a special way to the normal system of algebraic equations with approximate initial data; the parametric regularizing operator is built for a stable solving of the obtained normal system - this operator, in case of an appropriate parameter choice (which is called the Tikhonov regularization parameter), guarantees the stability of the approximate solution found for the normal system; the fairly simple to implement and efficient algorithm of finding the regularization parameter is offered for the constructed regularizing operator; a closed formula determines the calculated structural design parameters weights of the technical system and, thus, the effectiveness rate of the technical system under study.

It should also be noted that the proposed approach eliminates one of the main drawbacks of a well-known and frequently used method of weighting coefficients (this method is the main method for multicriterion linear programming problems solving) - the accountability lack of the weighting coefficients dependence on the partial quality indicators values of the considered technical system.

\section{ACKNOWLEDGMENTS}

The present article was executed within the framework of the following two Research Projects: 1) The State Research Programme "NextIT": Next generation Information and Communication Technologies (for the first co-author); 2) The European Regional Development Fund Project No. 2014 / 0029 / 2DP / 2.1.1.1 / 14 / APIA / VIAA / 088: "Development of an experimental long flight distance unmanned aerial vehicle prototype for multi-purpose environmental monitoring" (for the first and the second co-authors).

\section{REFERENCES}

[1] M. A. Yastrebenetskii, and G. M. Ivanova, Reliability of Automated Control Systems for Engineering Processes. Moscow: Energoatomizdat, 1989.

[2] V. S. Avduyevsky, V. I. Kuznetsov, N. D. Kuznetsov, V. A. Melnikov, V. P. Mishin, V. F. Utkin, K. V. Frolov, B. V. Gnedenko, I. N. Kovalenko, and B. F. Lomov, Reliability and Efficiency: Methodology. Organization. Terminology. Moscow: Engineering Industry, 1986.

[3] A. N. Tikhonov, and V. Ya. Arsenin, Solutions of Ill-Posed Problems. Moscow: Science, 1986.

[4] B. V. Gnedenko, Yu. K. Belyayev, and A. D. Solovyov. Mathematical Methods of Reliability Theory. Principal Characteristics of Reliability and Statistical Analysis. Moscow: URSS, 2013. 
[5] I. A. Ushakov, Yu. K. Belyaev, V. A. Bogatirov, and V. V. Bolotin, Reliability of Thechnical Systems. Moscow: Radio and Communication, 1985.

[6] V. G. Totsenko, Methods and Systems for Decision-Making Support. Kiyiv: Naukovo Dumka, 2002.

[7] V. V. Rykov, and V. Yu. Itkin. Mathematical Statistics and Planning an Experiment. - Moscow: Press of The Gubkin Russian State University of Oil and Gas, 2009.

[8] J. Antony, Design of Experiments for Engineers and Scientists. London: Elsevier Science \& Technology Books, 2003.

[9] R. L. Mason, R. F. Gunts, and J. L. Hess, Statistical Design and Analysis of Experiments with Applications to Engineering and Science. New Jersey, USA: WILEY-Interscience, 2003.

[10] S. Ch. Albright, W. Winston, and Ch. Zappe, Data Analysis and Decision Making. Mason, USA: South-Western Cengage Learning, 2010

[11] P. P. Biemer, and L. E. Lyberg, Introduction to Survey Quality. New Jersey, USA: Wiley-Interscience, 2003.

[12] C. Hwang, and F. C-.H. Rhee, "Uncertain fuzzy clustering: interval type-2 fuzzy approach to c-means." IEEE Transaction on Fuzzy Systems, Vol. 15, Issue 1, pp. 107-120, 2007.

[13] S. M. Yunusov, V. P. Labendik, and Sh. E. Guseynov, Monitoring and Diagnostics of Aircraft Gas Turbine Engines: Improvement of Models and Methods for Diagnosis of Gas Path of Gas Turbine Engines. Saarbrücken, Germany: Lambert Academic Publishing, 2014.
[14] S. A. Andreyev, and Sh. E. Guseynov, Regularizing Algorithms for Diagnosing: Applied to Gas Turbine Engines in Operation. Saarbrücken, Germany: Lambert Academic Publishing, 2013.

[15] V. K. Ivanov, "About linear ill-posed problems." Herald of The USSR Academy of Sciences, Vol. 145, Issue 2, pp. 270272, 1962.

[16] V. K. Ivanov, "About ill-posed assigned problems." Mathematical Collections, Vol. 61, Issue 2, pp. 211-213, 1963.

[17] Sh. E. Guseynov and V. I. Dmitriev, "Investigation of resolving power and solution detailedness of inverse problems of magnetotelluric sounding." Herald of the Lomonosov Moscow State University, Series 15: "Computational Mathematics and Cybernetics", Vol. 1, pp. 17-25, 1995.

[18] Sh. E. Guseynov and M. Okruzhnova, "Choice of a quasioptimal regularization parameter for the first kind operator equations." Transport and Telecommunication, Vol. 6, Issue 3, pp. 471-486, 1995.

[19] Sh. E. Guseynov and S. M. Yunusov, "New regularizing approach to determining the influence coefficient matrix for gas-turbine engines." in Dynamical Systems, Differential Equations and Applications. Vol. I, American Institute of Mathematical Sciences (AIMS), 2011, pp. 614-623.

[20] V. A. Morozov, Regularization Methods for Ill-Posed Problems. Florida, USA: CRC Press, 1993. 